\title{
Acute onset concomitant esotropia: when is it a sign of serious neurological disease?
}

\author{
Creig S Hoyt, William V Good
}

The onset of concomitant strabismus in most cases occurs during early infancy or childhood. 1 The two major categories of early onset concomitant esotropia are accommodative and congenital (infantile). While some disagreement exists as to the appropriate treatment of these strabismic disorders, there is general consensus that these are not usually causally related to any serious underlying neurological pathology.

In contrast, the patient who presents with the acute onset of esotropia and diplopia should prompt a careful consideration of whether or not the strabismus is a sign of serious central nervous system pathology. First and foremost, one needs to establish if the deviation is concomitant or inconcomitant. An acute onset esotropia with inconcomitance must be considered to be the result of paresis of the lateral rectus muscle (abducens nerve) until proved otherwise. There is no doubt that the vast majority of neuropathic or myopathic causes of strabismus present as an inconcomitant deviation.

On the other hand, does concomitancy in an acute onset esotropia assure one of the benign underlying nature of the strabismus? Until recently most authorities would probably have agreed that acute onset esotropia that is concomitant without divergence insufficiency is benign in nature with no risk that it might be associated with intracranial pathology. ${ }^{2}$ Regrettably, it is now apparent that this simple algorithmic way of viewing acute onset esotropia, while valid in the vast majority of cases, is not appropriate in any single case since too many exceptions exist. Concomitancy in acute onset esotropia does not rule out the possibility of an underlying serious neurological condition. ${ }^{3}$

How, then, is the clinician to evaluate the patient with acute onset concomitant esotropia? Does every patient with this form of strabismus deserve a neuroradiological and/or neurological evaluation? If not, what features or findings in a case of acute onset concomitant esotropia would indicate that this patient is at particular risk? In order to answer these questions an overview of the types of acute onset concomitant esotropia is necessary.

\section{Isolated acute onset concomitant esotropia}

Although scattered reports of cases of acute onset esotropia can be found in the literature dating back over the past 100 years, ${ }^{4-8}$ it is the paper of Burian and Miller that is generally accepted to be the pivotal work in this area. ${ }^{9}$ In reviewing the literature and their own experience, these two authors presented a thesis that acute onset concomitant esotropia could be separated into three distinct categories based on their clinical features and aetiology. Common to all three categories was the acute onset, concomitancy, a relatively large angle of deviation, good binocular potential, and no underlying neurological disease.
TYPE I - ACUTE ONSET CONCOMITANT STRABISMUS FOLLOWING OCCLUSION ('SWAN-TYPE')

In many ways this is the best known but also the most confounding form of acute onset concomitant strabismus. This form of strabismus may occur after patching for therapeutic reasons or as the result of monocular or asymmetric visual loss. The resulting strabismus in children and young adults is usually in the form of an esotropia wherein adult exotropia predominates. In the often cited case of Swan a 9-year-old boy who was significantly hyperopic developed an esotropia after monocular patching. ${ }^{6}$ Surgery was necessary to re-establish motor and sensory fusion. Burian and Miller commented that it was easy to understand 'the occurrence of an esotropia if a patient who underwent the patching had an uncorrected hypermetropia'. This would be especially the case in the patient with a high accommodative convergence/accommodation ratio. Yet, not all patients in this category are found to be hyperopic.

Patients with no significant refractive error have also been described to develop an acute onset concomitant esotropia following acute occlusion therapy. ${ }^{910}$ Are these patients simply those who had a phoric deviation under control and occlusion provided the opportunity for the deviation to become manifest? The evidence to our mind is conflicting. Burian and Miller cite the sizeable number of adults who develop an exotropia after partial or total loss of vision in one eye as being evidence to suggest they could not all have significant pre-existing phorias that lead to the manifest deviation. ${ }^{9}$ On the other hand, in a recent study of 14 non-strabismic volunteers with normal stereopsis who were monocularly patched for 2 to 24 hours, the induced deviations were all less than 10 prism dioptres. ${ }^{11}$ It should be recalled that the deviation in all forms of acute onset concomitant esotropia are usually reasonably large. ${ }^{12}$ It would appear that occlusion of one eye or loss of vision in one eye even in the absence of a significant hypermetropic refractive error may precipitate acute onset concomitant esotropia in some children or young adults.

TYPE II - CONCOMITANT CONVERGENT STRABISMUS OF THE 'FRANCESCHETTI' TYPE

In this group of patients Burian and Miller describe an acute onset esotropia syndrome that at first might be intermittent but quickly became constant. ${ }^{9}$ The refractive error was usually a minimal amount of hypermetropia and no accommodative element was detected. Although they stated that no underlying cause for the strabismus could be found they did suggest that a physical or psychic shock might be the precipitating factor. Reports of this type of acute onset concomitant esotropia have described its occurrence in multiple siblings ${ }^{8}$ as well as monozygous 
twins. ${ }^{13}$ Surgery has been required to re-establish ocular alignment in all patients thus far described.

The pathogenesis of the esotropia in this category is entirely unclear. However, it should be noted that not all patients described in the category adhered to the rigid definition of Burian and Miller. ${ }^{9}$ For example, at least one of the patients described by Noble and Malbran ${ }^{8}$ had at least a partial accommodative component to the deviation with a high accommodative convergence/accommodation ratio. Of more concern are the recent reports that challenge the thesis that the prognosis for re-establishing good binocularity in this group of patients is assured. ${ }^{1415}$ Heretofore, the good binocular potential of these patients has been a fundamental defining characteristic. This has been challenged by the recent work of Ohtsuki ${ }^{15}$ and co-workers and Boergen and Lorenz. ${ }^{14} \mathrm{~A}$ careful reading of these studies suggests that at least two groups of patients have been included in this single category. One group probably has a pre-existing phoria or microtropia with subnormal binocular vision before the onset of the tropia. This is supported by the findings of Crone who found a typical esophoria with microanomalous retinal correspondence in some patients with acute onset esotropia of the type II Franceschetti type using examination of the fixation disparity curve which did not coincide with the esophoria in those patents who had a normal sensory state. ${ }^{16}$ The other group of patients have undoubtedly enjoyed an entirely normal binocular status before the acute onset of the strabismus and have, therefore, an excellent prognosis for retaining binocularity no matter what interval of time separates the onset of esotropia and surgery. ${ }^{15}$ This latter group is the typical group originally described by Burian and Miller. ${ }^{9}$ We shall return to this point regarding the prognosis for re-establishing binocularity in acute onset concomitant esotropia when discussing its relation in patients with brain tumours.

TYPE III - CONCOMITANT CONVERGENT STRABISMUS OF THE 'BIELSCHOWSKY TYPE'

In 1922 Bielschowsky reported on patients with acute onset concomitant esotropia associated with myopia. ${ }^{4}$ Similar cases had been described previously by von Graefe. ${ }^{17}$ The consistent features of this group of patients were a myopia of 5 dioptres or less, esotropia at distance but maintained fusion at near, and no evidence of lateral rectus paralysis. Since Bielschowsky's original report, the characteristics have been redefined to include higher levels of myopia and constant deviations at both near and distance fixations. ${ }^{18}$ Subsequent reports have emphasised that good binocular function may be maintained in these patients with prisms. ${ }^{19}$ It should be noted, however, that these patients often present with a reasonably small angle of esotropia (10 prism dioptres or less), but gradually over a period of time develop an increasingly large angle that may preclude the use of prisms in treatment. Fortunately surgery is usually efficacious in re-establishing good binocular function in these patients.

Bielschowsky had no doubt that the myopia in these patients played a central role in the aetiology of this form of acute onset concomitant esotropia. Like von Graefe he assumed that the uncorrected myope tends to hold print excessively close to the eyes thus resulting in an inability to maintain a balance between the converging and diverging forces of the eye. Recently this theory to explain the pathogenesis of the strabismus in these patients, which primarily invokes increased tonus in the medial rectus muscles as being causative, has been challenged by a report that suggests that direct damage to the lateral rectus muscle may be the primary pathology in these cases. ${ }^{18}$
It should be apparent from the above summary that of the three categories of acute onset concomitant esotropia described by Burian and Miller only type II is likely to perplex the clinician with regard to its appropriate evaluation. Type I patients have a history of monocular occlusion or visual loss and type III patients will have a myopic refraction. Type II patients, on the other hand, are the real worry. How does one distinguish them from the uncommon but worrying cases of acute onset concomitant esotropia associated with serious neurological disease?

\section{Neurological disorders and concomitant esotropia}

That an acute onset concomitant esotropia may be associated with a brain tumour has been recognised with increasing frequency. ${ }^{20-27}$ Even those who studied acute onset concomitant esotropic patients without neurological disease have agonised about when neurological evaluation should be undertaken in this patient group. ${ }^{24}$ No single type of brain tumour or site within the brain can account for all the cases described thus far. Undoubtedly more than one aetiological mechanism is at play on this heterogeneous group of cases. Nevertheless a review of these reports provides a framework in which to consider the diagnostic dilemma that these patients present.

Anderson and Lubow described a 6-year-old boy with an acute onset concomitant esotropia associated with normal ductions and versions who was found to have papilloedema. ${ }^{22}$ Neuroradiographic studies documented hydrocephalus caused by a cystic lesion of the corpus callosum. Biopsy of the lesion revealed a poorly differentiated glioma. It is noteworthy that the patient's esotropia spontaneously resolved before any surgery or radiotherapy. It is tempting to suggest that this patient's esotropia, although concomitant, was the result of abducens nerve dysfunction in association with hydrocephalus. In this regard, Flynn's report on an 11-year-old girl who presented with an acute onset concomitant esotropia is enlightening. ${ }^{24}$ This patient subsequently developed, 6 weeks later, facial and abducens nerve palsies at which time the diagnosis of pontine glioma was established by appropriate neuroradiographic studies. Clearly this patient's initial concomitant esotropia was an early sign of the abducens nerve palsy that ultimately became apparent. In discussing this case, Jampolsky noted that the small $\mathrm{V}$ pattern seen on the initial examination when the deviation was still concomitant should have suggested the possibility that the primary problem was a paresis of the abducens nerve. ${ }^{28}$

Not all patients with acute onset concomitant esotropia associated with a brain tumour can be accounted for by the paretic mechanism, however. Zweifach reported the case of a 9-year-old boy who developed intermittent diplopia followed by a concomitant constant esotropia. ${ }^{23}$ The patient had no neurological symptoms or signs when first examined, but 28 months later when he developed signs of posterior fossa dysfunction he was investigated and found to have a medulloblastoma involving the cerebellum. At no time, over this long course did the esotropia become inconcomitant or exhibit other signs of paresis of the abducens nerve.

Is hydrocephalus, and not the brain tumour itself the central cause of the esotropia in these patients? The association of concomitant esotropia and hydrocephalus has been discussed in great detail. ${ }^{29-31}$ Harcourt should be credited for calling our attention to the frequent association of concomitant esotropia, as well inconcomitant esotropia, with the hydrocephalic patient. ${ }^{31}$ Moreover, he challenged the notion that the frequently seen concomitant esotropia in this setting was merely an expression of abducens nerve dysfunction. Hoyt and Daroff reported a case of a 3-monthold infant with intermittent hydrocephalus who developed 
chronic downward deviation of the eyes and acute onset concomitant esotropia each time the intraventricular pressure became elevated. ${ }^{32}$ These signs would completely resolve with restoration of normal intraventricular pressure. Furthermore at no time was abducens paresis noted. The association of acute onset concomitant esotropia in the Arnold-Chiari syndrome has been attributed to the co-existing hydrocephalus. ${ }^{12933}$ That the esotropia in this situation is related primarily to abducens nerve dysfunction seems unlikely in light of the longstanding concomitancy and the high frequency of an A rather than a $\mathrm{V}$ pattern. ${ }^{30}$ It seems irrefutable, therefore, that the relation between hydrocephalus and esotropia goes beyond the simplistic notion that all cases represent abducens nerve dysfunction.

Yet many of the patients with brain tumour and acute onset concomitant esotropia that have been reported do not show signs of either abducens nerve paresis or hydrocephalus. Only two of the six patients with brain tumour and acute onset concomitant esotropia described by Williams and Hoyt had elevated intracranial pressure. ${ }^{3}$ None of their cases developed signs of abducens nerve dysfunction. It is noteworthy that three of the six patients had bilateral abduction nystagmus. This finding has been discussed in more detail by Ciancia who examined eight patients who had posterior fossa tumours and acute onset concomitant esotropia. ${ }^{26}$ Seven of these patients exhibited abduction nystagmus and six were found to have asymmetric optokinetic nystagmus. Ciancia suggested that perhaps brainstem or cerebellar dysfunction alone could account for the concomitant esotropia. A similar thesis has been articulated by Lennerstrand who went so far as to suggest that vergence mechanisms in the mesencephalon may be especially important in the genesis of concomitant esotropia even in infantile esotropia. ${ }^{30}$ This is an appealing hypothesis for at least two reasons. (1) Primate models have identified cells in the midbrain that respond to pure vergence signals and are independent of versional eye movement. ${ }^{34}$ That these cells are both convergent and divergent responsive is especially noteworthy. (2) Acute onset concomitant esotropia with lesions of the caudal thalamus may reflect a disturbance of vergence inputs owing to extension of the pathology into the midbrain. ${ }^{35-37}$ Indeed the study of Kahara and coworkers demonstrated that esotropia occurred only in patients with extension of the lesion into the midbrain. ${ }^{37}$

While a precise understanding of the pathogenesis of acute onset concomitant esotropia seen in some patients with brain tumours has yet to be determined, it is clear that neither abducens nerve dysfunction nor hydrocephalus alone can account for all of the cases. From a practical point of view tumours of the cerebellum, brainstem, pituitary region, and corpus callosum have all been reported to be associated with acute onset esotropia. How is the physician to distinguish these patients from those with type II concomitant convergent strabismus of the 'Franceschetti type'. Obviously, any symptom or finding, such as headache, papilloedema, clumsiness, etc, would suggest that neurological investigation is warranted. Yet, is there anything about the ocular motor findings in these patients that might define these different groups? We would like to emphasise two points: (1) associated nystagmus - nystagmus of any kind is conspicuous by its absence in all reports of type II concomitant convergent strabismus of the Franschetti type. (2) In contrast, recall the importance of nystagmus in abduction in the cases with brain tumour reported by both Williams and $\mathrm{Hoyt}^{3}$ and Ciancia. ${ }^{26}$ This point has previously been discussed by Scott ${ }^{25}$ and cannot be overemphasised. That is, in evaluating any patient with acute onset concomitant esotropia, the detection of any nystagmus should be sufficient to prompt a neurological and neuroradiological investigation of the patient.

\section{Prognosis for restoration of binocularity}

A fundamental defining characteristic of type II concomitant convergent strabismus of the Franceschetti type is its excellent potential for re-establishing motor and sensory fusion. ${ }^{9}$ The reports of Boergen and Lorenz ${ }^{14}$ and Ohtsuki and co-workers ${ }^{15}$ should not be interpreted to the contrary. Both of these studies include patients who had pre-existing phorias or microtropias with poor stereo acuity. These patients need to be distinguished from the vast majority of cases of type II concomitant convergent strabismus in which excellent pre-existing binocularity was present.

Both Williams and $\mathrm{Hoyt}^{3}$ and Ciancia ${ }^{26}$ report the failure of re-establishing binocularity in their patients with brain tumours. Ciancia went so far as to detail the evaluation of a boy with a medulloblastoma of the cerebellum and acute onset of concomitant esotropia who 'when examined with prisms or the synoptophore was able to superimpose diplopic images with stereopsis perception, but was unable to maintain the images superimposed and had no motor fusion'. ${ }^{26}$ If a patient with acute onset concomitant esotropia cannot demonstrate motor fusion when examined with prisms or the synoptophore the possibility of an underlying neurological disease should be suspected.

It should be noted that the above discussion of acute onset concomitant esotropia in association with neurological disease is not complete. For example, myasthenia gravis may present with acute concomitant strabismus with no evidence of paresis. ${ }^{38} 39$ Eventually, however, the presence of ptosis, other signs of weakness, or change in the pattern of strabismus will suggest the appropriate diagnosis. The goal of this paper has been to outline the differences in patients with acute onset concomitant esotropia with coexisting central nervous system pathology from those who are entirely healthy.

\section{Conclusion}

Acute onset of concomitant esotropia is an uncommon form of strabismus. In the vast majority of cases it will have no obvious underlying neurological cause. When associated with a history of previous strabismus, occlusion therapy or monocular visual loss, or myopia it need cause little worry for the clinician. However, in the case of the patient with no apparent cause for the acute concomitant esotropia the possibility of an underlying neurological disease should at least be considered. Although no single clinical test appears to be able to identify the patient with neurological disease, the presence of nystagmus or the inability to restore binocularity in any of these patients should be considered sufficiently abnormal as to warrant neurological investigation.

A 702, 400 Parnassus Avenue,

CREIG $S$ HOYT

University of California,

San Francisco,

CA 94143, USA

Cincinnati, Ohio

WILLIAM V GOOD

1 Burian-von Noorden GK. Binocular vision and ocular motility. St Louis: CV Mosby, 1990: 389-90

2 Watson AP, Fielder AR. Sudden onset squint. Develop Med Child Neurol 1987; 29: 207-11.

3 Williams AS, Hoyt CS. Acute comitant esotropia in children with brain tumors. Arch Ophthalmol 1989; 107: 376-8.

4 Bielschowsky A. Das Einwartfschien der Myopia. Der Deutsche Ophthalmol Gesell 1892; 43: 245-8.

5 Burian HN. Motility clinic: sudden onset of comitant convergent strabismus. Am F Ophthalmol 1945; 28: 407-10. 
6 Swan KC. Esotropia following occlusion. Arch Ophthalmol 1947; 36: 444-51.

Franceschetti A. Le strabise conconcomitant aigu. Ophthalmologica 1952; 123: 219-26.

8 Norbis AL, Malbran E. Conconcomitant esotropia of late onset - pathological report in four cases in siblings. Br f Ophthalmol 1956; 40: 373-8.

9 Burian HM, Miller JE. Comitant convergent strabismus with acute onset. Am f Ophthalmol 1958; 45: 55-63.

10 Elahatton K, Repka MK. Prism treatment of acute esotropia following interruption of fusion. Am Orthoptic $\mathcal{F} 1994$; 44: 76-9.

11 Holmes JM, Katz KM. Recovery of phorias following monocular occlusion. I Pediatr Ophthalmol Strabismus 1994; 31: 10-4.

12 Goldman HD, Nelson LB. Acute acquired comitant esotropia. Ann Ophthalmol 1985; 17: 777-8.

13 Ahmed S, Young JDH. Late onset esotropia in monozygous twins. Br $\mathfrak{F}$ Ophthalmol 1993; 77: 189-91.

14 Boergen KP, Lorenz Z. Normosesoriches Spatschielen. Operation Zeitpunkt Und Binokularprognoses. Z Augenheilkd 1991; 12: 331-6.

15 Ohtsuki H, Hasebe S, Kobashi R, Okana M, Furese T. Critical period for restoration of normal stereo acuity in acute-onset esotropia. $A m \mathcal{F}$ Ophthalmol 1994; 118: 502-8.

16 Crone RA. Acute convergent strabismus. Diplopia. Amsterdam: Excerpta Medica, 1973: 184-6.

17 von Graefe A. Uber di bon Myopie abhangige Form Convergierenden Schielens und derheiong. Arch Ophthalmol 1864; 10: 156-75.

18 Meyer E. End stage fibrosis of the lateral rectus muscle in myopia with esotropia. Ophthalmic Res 1990; 29: 259-63.

19 Weber E. Mit Diplopie einhergehendes Konvergenzchielen Myoper (Bielschowski). Ophthalmologica 1947; 1114: 320-4.

$20 \mathrm{Wybar} \mathrm{K}$. The significance of squint in certain forms of malignant disease. Br Orthoptic f 1967; 24: 47-52.

21 Micketavage RC. Ophthalmologic disease presenting as orthoptic problems. Am Orthoptic f 1972; 22: 44-6.

22 Anderson WD, Lubow M. Astrocytoma of the corpus collosum presenting with acute comitant esotropia. Am $\mathcal{f}$ Ophthalmol 1970; 69: 594-8.

23 Zweifach PH. Childhood esotropia with delayed appearance of cerebellar tumor. Neuro-Ophthalmology 1981; 1: 291-3.
24 Flynn JT. Problems in strabismus management. Transactions New Orleans Academy of Ophthalmology. New York: Raven Press, 1986: 456.

25 Scott W. Problems in strabismus management. Transactions New Orleans Academy of Ophthalmology. New York: Raven Press, 1986: 456.

26 Ciancia AO. Bielschowsky lecture. On infantile esotropia with nystagmus in abduction. $\mathcal{f}$ Pediatr Ophthalmol Strabismus (in press).

27 Joy $\mathrm{AE}$, Rosales $\mathrm{T}$. Accommodative esotropia in children with brain tumor. Ophthalmology 1994; 101 (suppl): 115.

28 Jampolsky AJ. Problems in strabismus management. Transactions Ner Orleans Academy of Ophthalmology. New York: Raven Press, 1986: 456.

29 Bigland AW. Ophthalmologic complications of meningomyelocele: a longitudinal study. Trans Am Ophthalmol Soc 1990; 88: 389-459.

30 Lennerstrand B, Gallo FE, Samuleson L. Neuro-ophthalmologic findings in relation to CNS lesions in patients with meningomyelocele. Develop Med Child Neurol 1990; 32: 423-31.

31 Harcourt RB. Ophthalmologic complications of melingomyelocele and hydrocephalus in children. Br f Ophthalmol 1968; 52: 670-6.

32 Hoyt WF, Daroff RB. Supernuclear disorders of ocular control systems in man. In: Bach-Y-Rita Collins CC, eds. The control of eye movements. New York: Academic Press, 1971: 199.

33 Passo M, Schults WT, Talbot T. Acquired esotropia: a manifestation of Chiari I. Malformation. $\mathcal{f}$ Clin Neuro-Ophthalmol 1984; 4: 151-4.

34 Judge J, Cummings BG. Neurons in the monkey midbrain with activity related divergence eye movements and accommodation. 7 Neurophysiol 1986; 55: 915-30.

35 Hertle RW, Bienfang DC. Oculographic analysis of acute esotropia secondary to a thalamic hemorrhage. $f$ Clin Neuro-Ophthalmol 1990; 10: 21-6.

36 Gomez CR, Gomez SM, Selhorst JB. Acute thalamic esotropia. Neurology 1988; 38: 1759-62

37 Kahara N, Fato K, Muraki M, Tanka K, Kaneko M. CT classification of small thalamic hemorrhages and their clinical significance. Neurology 1986; 36: 165-72.

38 Davidson JL, Rosenbaum AL, McCall LC. Strabismus surgery in patients with myasthenia. $\mathcal{F}$ Pediatr Ophthalmol Strabismus 1993; 30: 292-5.

39 Moore S, Welter P. Ophthalmologic diagnosis and evaluation of prism therapy for ocular myasthenia. Am Orthoptic f 1993; 43: 97-101. 